\title{
Wellness Review 2021, Part 1
}

\author{
Martin Huecker, $\mathrm{MD}^{1 *}$, Brian Ferguson, DO ${ }^{1,2}$, Jacob Shreffler, $\mathrm{PhD}^{1}$
}

\begin{abstract}
Introduction: This article presents a curated selection of the wellness literature from January to June of 2021. JWellness editors offer a summary of recent publications within the wellness domain to seek an understanding of both burnout prevention and, more importantly, thriving in the medical profession.

Methods: For the interval of Jan 1 to June 30, 2021, a UofL librarian queried PubMed for empirical research studies, review articles, and editorials related to healthcare professional wellness. Excluding papers related to COVID-19 (due to extensive prior coverage) and editorials/commentaries, the editors narrowed to 43 articles (systematic reviews, meta-analyses, general reviews, and clinical trials) for this review.

Literature in Review: Six chief themes emerged: general wellness / burnout, technology, training, nursing professional wellness, mindfulness and resilience, and physician specialty-specific considerations. We note the predominance of descriptive articles, though this round included more clinical trials and reviews of clinical trials. Overall quality of evidence remains low, however the conglomerate multinational dataset is expanding rapidly. Innovations in electronic documentation, early educational interventions, and diverse studies from various medical specialties converge to provide insight into a state of thriving among healthcare professionals.

Conclusion: Current literature into healthcare professional wellness continues to describe burnout, but increasingly includes dedicated wellness interventions. Resilience and mindfulness training work, but system level interventions must accompany efforts. All medical specialties are affected, and the continuing conversation should include healthcare executives and other leadership.
\end{abstract}

DOI: 10.18297/jwellness/vol3/ iss $2 / 7$

Publication Date: Sept 8, 2021

Website: https://ir.library.louisville.edu/jwellness/

Recommended Citation: Huecker M, Ferguson B, Shreffler J. (2021) "Wellness Review 2021, Part 1," Journal of Wellness:

Vol. 3 : Iss. 2, Article 7.

Affiliations: : 1 University of Louisville, Department of Emergency Medicine, ${ }^{2}$ AFSOC (Air Force Special Operations Command)

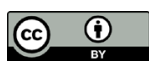

\section{INTRODUCTION}

Welcome to Part 1 of the Journal of Wellness review of 2021 wellness literature. In this review, editors continue the goal of bringing awareness of recent relevant publications on healthcare professional wellness. We summarize new science and resilience initiatives published outside of JWellness that seek understanding of burnout and its prevention, with emphasis on research into thriving in physicians, nurses, and all other healthcare professionals.

\section{METHODS}

For the interval of Jan 1 to June 30, 2021, a UofL librarian queried PubMed for articles related to healthcare professional wellness. An initial PubMed search for articles related to "burnout," "wellness," "well-being," and "resilience" among healthcare workers published in English between January 1 and July 1 of 2021 yielded 2,084 results. The "Publication Type" filter was used to limit the results to reviews, systematic reviews, meta-analyses, randomized controlled trials, and clinical trials, to yield a set of 324 results for initial screening.

Due to extensive prior coverage, we removed articles related to COVID-19 (84). Editors MH and JS reviewed the remaining

${ }^{*}$ Correspondence To: Martin Huecker Email: martin.huecker@louisville.edu
240 papers, choosing those directly relevant to healthcare professional wellness, and removing articles deemed irrelevant. Cross-matching of selections yielded 35 total papers. Reviewing a rolling list of articles curated throughout 2021 by JWellness editors, we found eight not captured in the above Pubmed search, providing total of 43 for inclusion in this review.

\section{LITERATURE REVIEW}

Research into healthcare professional burnout and wellness continues to focus heavily on effects of COVID-19, even prior to the Delta variant and new waves of infection. After excluding COVID-19 coverage, we found several interesting themes surface. Admitting some overlap, the 43 articles neatly grouped into one of the following themes: general wellness / burnout, technology, training, nursing professional wellness, mindfulness and resilience, and physician specialty-specific considerations.

\section{General Wellness/Burnout}

Starting out in the late 70s, the search term burnout continues to increase in overall number of citations in Pubmed. After a brief drop in the early 2000s, we have seen a huge increase from

Copyright: $\odot 2021$ The author(s). This is an open access article distributed under the terms of the distribution, and reproduction in any medium, provided the original author and source are credited. 
about 2006 (60 per 100,000) until present (>180 per 100,000) (https://esperr.github.io/pubmed-by-year/?q1=burnout). Using the above search terms, including reviews and trials, excluding COVID, and removing the other articles described herein, we cover eight articles on general wellness and burnout in medical professionals.

In a systematic review on burnout, Balendran et al. looked solely at surgical specialists in the United Kingdom [1]. They found ten papers from 2000-2019 that found an overall burnout prevalence of $32 \%$. Trainees (younger age and lower clinical grade) were the most at risk with 59\% having burnout. Those married or living with a partner had some protection from burnout.

De Simone et al. performed a systematic review and meta-analysis on organizational strategies to reduce physician burnout [2]. Including 19 studies for full analysis, they found that organizational interventions had slightly larger aggregate reduction of burnout versus physician targeted interventions. Table 1 from the systematic review provides an excellent summary of all studies. The organizational interventions seemed to effectively combat depersonalization and lack of personal accomplishment.

In an interesting review in Health Policy and Planning, Russo et al. synthesized 50 years of research on how economic recessions affect healthcare workers [3]. They found 57 papers describing circumstances of six different recessions, though more than half of the papers related to 2010-2015 Europe. Overall, recessions seem to increase demand for health workers except in countries who adopted austerity measures. In North America, especially for nurses, no studies described a decrease in job opportunities for healthcare workers. Low-income countries suffered from loss of motivation, absenteeism, and abuse of health systems. This very interesting paper packed quite a lot of information in just 10 pages, with Table 1 and Figure 2 particularly enlightening.

Mangory et al. reviewed the literature on physician burnout and observed adverse patient outcomes [4]. Out of 11 articles in the final review, four found a clear link between burnout and adverse events, but the other six found no association. Overall, burnout less strongly correlated with patient adverse events (compared to depression - depressed residents made 6.2 times more errors than non-depressed residents). Lawrence et al. wrote a systematic review of racial/ethnic differences in medical student and physician burnout [5]. In sixteen studies, mostly cross-sectional and in North America, they found no clear overall difference in burnout by race / ethnicity, noting their results as inconclusive.

Uhlig-Reche found a cohort of 369 female attending physician runners, collecting data on work-life integration [6]. Burnout increased with more weekly work hours and with increased responsibility of "domestic duties." They concluded that despite healthy exercise habits, these physicians were at risk of burnout.

The final two papers of the general burnout category presented no data but do represent more voices asking to "shift the focus from burnout to wellness." Vercio et al. point out the limitations of well-meaning but often ineffective wellness curricula, especially during stresses such as a global pandemic [7]. They argue that we should embrace (inevitable) adversity and work on resilience training while also cultivating resilient organizations that more effectively connect with their providers.

Bradford et al. write in an OB/GYN journal on the somewhat unrealistic expectation of the "physician, heal thyself" Biblical proverb [8]. The Oath of Geneva says, "THE HEALTH AND WELL-BEING OF MY PATIENT will be my first consideration" but also says "I WILL ATTEND TO my own health, well-being, and abilities in order to provide care of the highest standard." Though not mutually exclusive, these two goals are challenging to accomplish simultaneously. Authors look ahead to efforts such as the quadruple aim (adding clinician experience to the accepted aims of patient experience, population health, and low-cost care) and broad efforts by the National Academy of Medicine's Action Collaborative on Clinical Well-Being and Resilience.

\section{Technology}

Five interesting articles filtered out in the first half of 2021 covered technology and healthcare professional wellness. Lorkowski et al. discussed the "life cycle of documentation" [9]. They explain the illogical lifespan and new forms of Electronic Health Records (EHRs) that we suffer through, a situation inferior to an iterative / evolutionary model. This "mass computerization" clearly leads to burnout via poor user-friendliness of software and hardware that literally sit in the way as we care for patients. Nguyen et al. performed a systematic review of causes of and solutions to EHR obstacles to wellness [10]. Narrowing down to 35 included articles, they found many predictors of EHR trouble: total EHR time, after-hours EHR time, lack of on-site EHR support, poor perceived EHR usability, in-basket burden, and documentation burden. Physicians across the board cited these issues as areas for potential improvement. "As primary stakeholders, physicians should be included in the planning and implementation of such modifications to ensure compatibility with physician needs and clinical workflows" [10].

Thomas Craig et al. wrote a systematic review including 81 studies of organizational interventions to specifically mitigate physician burnout [11]. An impressive $68 \%$ of them reported improvement in burnout measures, using many strategies: optimizing technology, training, less documentation and task time, expanding the care team, and QI processes in workflows. Every chief executive office, chief physician executive, and department chair should read this article thoroughly for several high yield ideas. Virone et al. looked at digitization bias and work-related "technostress [12]." The evidence-based methods of coping with technostress look just like general measures well-known to improve job satisfaction: autonomy, competence, understanding of roles, time pressure, attitude, security and ergonomics.

Johnson et al. tackled the EHR elephant with a "story of three eras" (three different informatics eras that line up with "Berwisk's health system eras" [professional dominance, accountability and the upcoming moral]) [13]. This somewhat technical article appears in the Journal of the American Medical Informatics Association. The EHR eras include the Era of Enlightenment (antiquity to 1990s), Era of Entanglement (1990s-present), and Era of Engagement (2030 onward). This article looks more mechanistically at EHRs, rather than focusing 
on provider wellness. They also express optimism:

Perhaps the only constant in the history of medical records is their dynamism-in time, the frustration at clinical alerts or mandated points of data entry will give way to new successes and challenges. Just as the limitations of paper records once motivated the adoption of electronic systems, today's imposition of data entry onto clinicians has given discussions about the limitations of our EHRs a deep urgency. With new policy prescriptions and the careful collaboration of informaticians and clinicians, we see the Era of Engagement as holding the potential to remove much of that burden from clinical workflows, returning clinicians to the bedside without dissolving the positive qualities of the EHR.

\section{Training}

Seven publications present trials or reviews of wellness in physicians in training. Eskander et al. wrote an extremely valuable systematic review on wellness interventions in residents, sadly finding only 18 studies on 666 residents meeting their criteria for qualitative review (13 of 18 poor quality, mostly due to inconsistent wellness measures) [14]. Certain characteristics predicted success in enhancing well-being: peer support, individual meditation practices, those based on educational theory, those that used feedback from the resident subjects to guide design, those that incorporated the initiatives into established didactics (in contrast to extra lectures or modules), and those that had voluntary participants. Grech et al. provide a narrative review of how the educational environment affects burnout in residents [15]. Reviewing recent papers (2015-2020), they included 27 studies with a wide range of reported burnout (10\%-62\%). Educational environmental factors complicated burnout, with hours worked, mistreatment, harassment and injustice leading to poor outcomes.

Shutt et al. in Academic Medicine provide a large dataset on wellness programs in US and Canadian medical schools [16]. They received responses from 105 of 159 (65\%) schools, noting that $93 \%$ had formal wellness programs (potential for selection bias). Schools had a mix of preventive, reactive, and cultural programming, with most lacking space devoted to structural programming. Here are their definitions: "Preventative programming proactively addresses stressors of medical education. Reactive programming connects distressed students to support resources. Structural programming addresses the curriculum or learning environment. Cultural programming addresses institutional and student perspectives on wellness programming." The most reported obstacles included lack of financial/administrative support and lack of time. Sixty-two percent of schools did evaluate their wellness programs. Most (83\%) assessed using the Association of American Medical Colleges Graduation Questionnaire, while $62 \%$ used their own annual surveys.

Seo et al. in BMC Medical Education composed a systematic review of resilience curricula in medical schools [17]. Twenty-one studies (only two RCTs) evaluated or described resilience curricula. The heterogeneity and lack of prior validation in duration, delivery, topics, and outcomes make this review difficult to trust as a synthesis. Most of the cited research boasted improvements in resilience of subjects, but three found worsened resilience at the end of training.

Investigators conducted a scoping review on literature examining spirituality and religion in resident training and clinical practice, finding 44 studies meeting inclusion criteria. The authors discovered that superior spiritual well-being of residents correlated with a greater sense of work accomplishment, overall health, and decreased burnout. Residents recognized the importance of addressing spirituality and religion in patient care, but note deficits in training on what to ask patients, time efficiency in clinical encounters, and how to manage personal discomfort [18].

Physicians from Stanford University describe the development of a physician wellness fellowship in emergency medicine. Implemented in 2019-2020, the fellowship aims to train experts to advance the topic of physician wellness. Authors present a reflective discussion describing the importance of institutional support and faculty expertise in physician wellness to initiate comparable programs. The authors intend this exposition to serve as a model for other programs, including those outside of EM, to develop experts in physician wellness [19].

One final article on physicians in training proposes integration of sleep knowledge in the standard curriculum for anesthesiology residency. The authors describe five central competency domains: sleep physiology, evaluation of sleep health, evaluation for sleep disorders and clinical implications, professional and academic roles, and "WELLness" ("SLEEP WELL"). They assert that physicians should have experience with these topics to establish collaborative networks (specifically between sleep medicine and anesthesiology), to open up possibilities for dual crediting among anesthesiologists, and to optimize sleep hygiene and physician wellness [20]. This unique article provides in depth content on sleep physiology, while philosophically integrating sleep health with overall wellness.

\section{Nursing profession}

The COVID-19 pandemic has exposed the dramatic vulnerability of healthcare systems worldwide related to supply and health of nurses. Five publications directly addressed wellness or burnout in nursing professionals, four reviews and one study. Jarden et al. reviewed 22 studies on psychological well-being and ill-being of nurses in their first year of practice. Social connection and support consistently facilitated feelings of value and team membership and reduced feelings of overwhelm, stress, loneliness, and inadequate preparation [21].

Jun et al. performed a systematic review of the impact that nurse burnout has on patient and organizational outcomes, finding 20 studies for inclusion. All five of their predetermined outcomes (patient safety and satisfaction, quality of care, nurses' organizational commitment, and productivity) varied inversely with the levels of nurse burnout [22]. Xie et al. performed a systematic review and meta-analysis of compassion satisfaction and compassion fatigue across 79 studies, including 28,509 nurses from 11 different countries [23]. "Compassion satisfaction is the sense of gratification that comes from providing care to relieve the suffering of other people ... compassion fatigue is viewed as emotional, physical and psychological exhaustion due to chronic work-related stress exposure among healthcare providers." The nurses most at risk for compassion fatigue were those working in intensive care and those practicing in Asian regions. According to pooled mean scores, compassion 
satisfaction was highest in 2015, while burnout and secondary traumatic stress were highest in 2019.

Wei et al. cover the impact of nursing student burnout on psychological well-being and academic achievement [24]. Not surprisingly, burnout had a negative relationship with self-concept and engagement. The authors did describe risk factors for burnout (financial strain, adverse childhood experiences, higher negative affectivity, sense of coherence, resilience, etc.), and devoted space to successful interventions to reduce nurse burnout (mindfulness training and acceptance and commitment training $[\mathrm{ACT}])$.

An interesting study by Sasaki et al. used a smartphone app (ABC Stress Management, apparently not available for download in app stores) with basic cognitive behavioral therapy to assess impact on work engagement in 949 nurses over 7 months [25]. The 6-week, 6-lesson plan was offered in free-choice or fixed order, with a (third) control group. At 3 months, only the fixed order group showed a benefit in engagement, and at 7 months neither of the intervention groups still had the benefit. Oddly, the control group had steady improvement in work engagement throughout the study.

\section{Mindfulness and Resilience}

One empirical article examined physicians' circadian rhythm chronotype, or morning-eveningness (ME) preference for daily duties and sleep timing. The authors described three natures: morning-type ("early birds") neural-type (individuals more in the middle), and evening-type ("night owls"). The authors discovered that morning-type individuals who worked morning shifts and evening-type individuals who worked evening shifts had higher levels of job satisfaction than those mismatched (controlling for income, age, marital and employment status) [26].

Numerous reviews were published in the first half of 2021 around physician mindfulness. A systematic review and meta-analysis focused on the effects of mindfulness interventions to reduce burnout and stress among providers. The authors found 25 studies meeting inclusion criteria. Overall, mindfulness interventions had small but significant reductions in burnout and "medium" reductions in stress. The authors concluded that mindfulness interventions can effectively reduce stress and burnout; but they recommend future studies with long-term data to determine optimal interventions [27].

Another systematic review focused on the relationship between emotional intelligence and mindfulness. Aggregating 10 studies, authors found a positive correlation between emotional intelligence and mindfulness - specifically, mindfulness increased the ability to regulate emotions. The authors describe how mindfulness interventions promote positive aspects of emotional intelligence and reduce emotional exhaustion [28]. Another review on mindfulness focused on healthcare professionals and medical students. The authors covered recent (up to July 2019) mindfulness literature and detailed how self-awareness in providers and medical students may allow them to engage in self-care and better equip them to manage stress [29].

An educational review paper described "gratitude, acceptance, intention, and nonjudgment" (GAIN) and how to mitigate burnout in medicine. The author detailed the issues surrounding burnout and proposed strategies to implement the practice of mindfulness with a specific focus on GAIN, which may result in physicians leading more resilient and happier lives [30].

A systematic review described cognitive flow in healthcare settings. The authors assert that cognitive flow experience can enhance wellness through augmenting meaning in daily exertions. Reviewing the prior literature, authors conclude that the experience of cognitive flow may safeguard individuals from burnout and stress. Individualized training and system-level interventions should be utilized to optimize cognitive flow in the work setting [31].

A meta-analysis aimed to better understand resilience interventions in physicians, finding 11 studies that met inclusion criteria. Interventions for resilience among providers were associated with significant benefits; longer (greater than a week) and emotional-supporting-coping (compared to mindfulness-meditation-relaxation) interventions led to more optimal outcomes [32].

One systematic review focused on available resources for second victims, in this case physicians harmed by an adverse event. The authors found scant descriptions of strategies to address this phenomenon outside of the U.S. They found hindrances to implementation of resources for second victims, such as blame culture, insufficient resources, and providers choosing not to seek necessary help. Organizations should invest in second victim support resources to help physicians become more resilient [33].

Kletter et al. reviewed positive psychology interventions within healthcare workers, finding 29 studies with widely varied outcome measures and mostly low-quality evidence. While few study outcomes showed statistical significance, many found trends associated with positive psychology's impact on healthcare providers. Furthermore, the authors postulate a logic model for future interventions related to positive psychology in healthcare providers [34].

\section{Physician Specialties}

Researchers studied mood alterations in 52 pediatric emergency physicians because of social, behavioral, affective, and cognitive changes throughout clinical shifts. The authors found that repeating patient assessments, particularly early in a clinical shift, was associated with lower positive affect and that higher levels of self-capacity for care correlated with increased levels of positive affect. Furthermore, the authors found that residents, advanced practice providers, attending physicians and fellows demonstrated a negative trend in positive mood as the shift progressed [35].

A qualitative study investigated EM residents' perceptions regarding burnout. The authors utilized focus groups with residents and recent program graduates as well as curricular evaluation data to depict portrayals of burnout in EM physicians. Residents were impacted by burnout in their clinical experience and personal lives. As a group, they viewed burnout as an inevitable part of what they go through as physicians [36]. JWellness editors find this concerning, making the scope and research efforts of the journal even more crucial.

A review of burnout in neurosurgery identified 32 studies, 
with most utilizing the Maslach Burnout Inventory (MBI) as a measure. Burnout ranged from 11.2 to $67 \%$ among residents and 15 to $57 \%$ among attendings. Identified sources of burnout among physicians included poor operative experience, social stressors, financial concerns, and poor-work life balance [37].

One paper focused on the prevalence of OB-GYN provider burnout and strategies to counter it. The authors detailed high estimates of burnout in OB-GYN (40 to 75\%), with effects ranging from emotional exhaustion to suicidal ideation. Mitigation strategies outlined by the authors included optimizing health and fitness, social support, being assertive, "letting things go," and reflecting and taking control [38]. In another OB-GYN paper, authors selected twenty studies for a scoping review. They noted an overall lack of quality research but highlight research showing success with interventions such as yoga, nutritional programs, narrative medicine, and counseling or debriefing sessions. The established interventions were mainly focused on individual interventions, rather than structural changes [39].

One systematic review of the literature investigated burnout prevalence in palliative care providers and potential interventions to reduce these experiences. The authors found that burnout prevalence varied dramatically ( 3 to 66 percent), with those in general settings experiencing more burnout than those in specialized palliative care. Meditation, peer-coaching, and art-therapy reduced burnout [40].

One research team utilized two small-randomized trials to determine the effect of enhanced stress resilience training within surgeons. Participants in the first randomized study intervention group completed eight weekly, 2-hour mindfulness courses; the second intervention group completed six weekly, 90-minute classes with more focus on a career in surgery. Neither trial resulted in a significant difference between control and intervention groups in reduction of stress, but the pilot work demonstrated benefits of participation on cognitive function and mindfulness (found in study 1) and lower emotional exhaustion and depersonalization (found in both studies) [41].

A group of authors describe the central facets of wellness (physical, mental, emotional, and spiritual) and discuss how to implement strategies to optimize wellness in orthopedic surgeons. They include interesting pearls, such as how to avoid acute and chronic musculoskeletal injuries (table height, posture, floor mats and "intraoperative targeted stretch microbreaks") as well as sections on visualization, self-compassion, spiritual wellness, and the importance of community and connection [42].

Bingemann et al. discussed physician wellness in allergy and immunology, outlining the often amorphous idea, and challenging cultivation, of a "culture of wellness." Creating a culture of wellness requires deliberate choice of interventions and measures of wellness, involvement of leadership, attention to engagement, and confronting potential barriers [43].

\section{CONCLUSION}

JWellness editors express optimism regarding interventions and potential for burnout mitigation and resilience enhancement. Forward-thinking research on electronic health records presents potential solutions to logistical challenges. Publications addressing wellness and burnout in medical trainees continues to accumulate as medical schools across the country introduce wellness curricula aggressively and early. Diverse studies from various medical specialties seem to be converging on the interplay of systematic and individual efforts to move beyond burnout to flourishing for healthcare professionals.

\section{REFERENCES}

1. Balendran B, Bath MF, Awopetu AI, Kreckler SM. Burnout within UK surgical specialties: a systematic review. Ann R Coll Surg Engl. 2021 Jul;103(7):464-70.

2. De Simone S, Vargas M, Servillo G. Organizational strategies to reduce physician burnout: a systematic review and meta-analysis. Aging Clin Exp Res. 2021 Apr;33(4):883-94.

3. Russo G, Silva TJ, Gassasse Z, Filippon J, Rotulo A, Kondilis E. The impact of economic recessions on health workers: a systematic review and best-fit framework synthesis of the evidence from the last 50 years. Health Policy Plan. 2021 May;36(4):542-51.

4. Mangory KY, Ali LY, Rø KI, Tyssen R. Effect of burnout among physicians on observed adverse patient outcomes: a literature review. BMC Health Serv Res. 2021 Apr;21(1):369.

5. Lawrence JA, Davis BA, Corbette T, Hill EV, Williams DR, Reede JY. Racial/Ethnic Differences in Burnout: a Systematic Review. J Racial Ethn Health Disparities. 2021 Jan;•••:1-13. ; Epub ahead of print.

6. Uhlig-Reche H, Larson AR, Silver JK, Tenforde A, McQueen A, Verduzco-Gutierrez M. Investigation of work-life integration on burnout symptoms in women physician runners: a cross-sectional survey study. BMJ Open Sport Exerc Med. 2021 Feb;7(1):e001028.

7. Vercio C, Loo LK, Green M, Kim DI, Beck Dallaghan GL. Shifting Focus from Burnout and Wellness toward Individual and Organizational Resilience. Teach Learn Med. 2021 Feb;•••:1-9. ; Epub ahead of print.

8. Bradford L, Glaser G. Addressing Physician Burnout and Ensuring High-Quality Care of the Physician Workforce. Obstet Gynecol. 2021 Jan;137(1):3-11.

9. Lorkowski J, Maciejowska-Wilcock I, Pokorski M. Overload of Medical Documentation: A Disincentive for Healthcare Professionals. Adv Exp Med Biol. 2021;1324:1-10.

10. Nguyen OT, Jenkins NJ, Khanna N, Shah S, Gartland AJ, Turner K, et al. A systematic review of contributing factors of and solutions to electronic health record-related impacts on physician well-being. J Am Med Inform Assoc. 2021 Apr;28(5):974-84.

11. Thomas Craig KJ, Willis VC, Gruen D, Rhee K, Jackson GP. The burden of the digital environment: a systematic review on organization-directed workplace interventions to mitigate physician burnout. J Am Med Inform Assoc. 2021 Apr;28(5):985-97.

12. Virone C, Kremer L, Breil B. Which Factors of Digitisation Bias the Work-Related Stress of Healthcare Employees? A Systematic Review. Stud Health Technol 
Inform. 2021 May;281:916-20.

13. Johnson KB, Neuss MJ, Detmer DE. Electronic health records and clinician burnout: A story of three eras. J Am Med Inform Assoc. 2021 Apr;28(5):967-73.

14. Eskander J, Rajaguru PP, Greenberg PB. Evaluating Wellness Interventions for Resident Physicians: A Systematic Review. J Grad Med Educ. 2021 Feb;13(1):58-69.

15. Grech M. The Effect of the Educational Environment on the rate of Burnout among Postgraduate Medical Trainees - A Narrative Literature Review. J Med Educ Curric Dev. 2021 May;8:23821205211018700.

16. Schutt A, Chretien KC, Woodruff JN, Press VG, Vela M, Lee WW. National Survey of Wellness Programs in U.S. and Canadian Medical Schools. Acad Med. 2021 May;96(5):728-35.

17. Seo C, Corrado M, Fournier K, Bailey T, Haykal KA. Addressing the physician burnout epidemic with resilience curricula in medical education: a systematic review. BMC Med Educ. 2021 Feb;21(1):80.

18. Chow HH, Chew QH, Sim K. Spirituality and religion in residents and inter-relationships with clinical practice and residency training: a scoping review. BMJ Open. 2021 May;11(5):e044321.

19. Singh J, Poffenberger C, Smith-Coggins R. Creating leaders through scholarship: the development of a physician wellness fellowship in emergency medicine. AEM Educ Train. 2021 Aug;5(4):e10627.

20. Singh M, Gali B, Levine M, Strohl K, Auckley D. Integrating Sleep Knowledge Into the Anesthesiology Curriculum. Anesth Analg. 2021 May;132(5):1296-305.

21. Jarden RJ, Jarden A, Weiland TJ, Taylor G, Brockenshire N, Gerdtz M. Registered Nurses' experiences of psychological well-being and ill-being in their first year of practice: A qualitative meta-synthesis. J Adv Nurs. 2021 Mar;77(3):1172-87.

22. Jun J, Ojemeni MM, Kalamani R, Tong J, Crecelius ML. Relationship between nurse burnout, patient and organizational outcomes: systematic review. Int J Nurs Stud. 2021 Jul;119:103933.

23. Xie W, Chen L, Feng F, Okoli CT, Tang P, Zeng L, et al. The prevalence of compassion satisfaction and compassion fatigue among nurses: A systematic review and meta-analysis. Int J Nurs Stud. 2021 Aug;120:103973.

24. Wei H, Dorn A, Hutto H, Webb Corbett R, Haberstroh A, Larson K. Impacts of Nursing Student Burnout on Psychological Well-Being and Academic Achievement. J Nurs Educ. 2021 Jul;60(7):369-76.

25. Sasaki N, Imamura K, Tran TT, Nguyen HT, Kuribayashi K, Sakuraya A, et al. Effects of Smartphone-Based Stress Management on Improving Work Engagement Among Nurses in Vietnam: Secondary Analysis of a Three-Arm Randomized Controlled Trial. J Med Internet Res. 2021 Feb;23(2):e20445.

26. Amini F, Moosavi SM, Rafaiee R, Nadi Ghara A, Babakhanian M. Chronotype patterns associated with job satisfaction of shift working healthcare providers. Chronobiol Int. 2021 Apr;38(4):526-33.

27. Fendel JC, Bürkle JJ, Göritz AS. Mindfulness-Based
Interventions to Reduce Burnout and Stress in Physicians: A Systematic Review and Meta-Analysis. Acad Med. 2021 May;96(5):751-64.

28. Jiménez-Picón N, Romero-Martín M, Ponce-Blandón JA, Ramirez-Baena L, Palomo-Lara JC, Gómez-Salgado J. The Relationship between Mindfulness and Emotional Intelligence as a Protective Factor for Healthcare Professionals: systematic Review. Int J Environ Res Public Health. 2021 May;18(10):5491.

29. Chmielewski J, Łoś K, Łuczyński W. Mindfulness in healthcare professionals and medical education. Int J Occup Med Environ Health. 2021 Jan;34(1):1-14.

30. Hammer GB. Mindfulness and GAIN: the solution to burnout in medicine? Paediatr Anaesth. 2021 Jan;31(1):74-9.

31. McQueen S, Jiang S, McParland A, Hammond Mobilio M, Moulton CA. Cognitive flow in health care settings: A systematic review. Med Educ. 2021 Jul;55(7):782-94.

32. Angelopoulou P, Panagopoulou E. Resilience interventions in physicians: A systematic review and meta-analysis. Appl Psychol Health Well-Being. 2021 May;aphw.12287.

33. Busch IM, Moretti F, Campagna I, Benoni R, Tardivo S, Wu AW, et al. Promoting the Psychological Well-Being of Healthcare Providers Facing the Burden of Adverse Events: A Systematic Review of Second Victim Support Resources. Int J Environ Res Public Health. 2021 May;18(10):5080.

34. Kletter M, Harris B, Brown C. Outcomes, mechanisms and contextual factors of positive psychology interventions for health workers: a systematic review of global evidence. Hum Resour Health. 2021 Feb;19(1):24.

35. Assaf RR, Pham PK, Schmidt AR, Gorab A, Chang TP, Liu DR. Pediatric emergency department shift experiences and moods: an exploratory sequential mixed-methods study. AEM Educ Train. 2021 Mar;5(3):e10572.

36. Jain A, Tabatabai R, Vo A, Riddell J. "I Have Nothing Else to Give": A Qualitative Exploration of Emergency Medicine Residents' Perceptions of Burnout. Teach Learn Med. 2021 Jan;33(4):1-9.

37. Mackel CE, Nelton EB, Reynolds RM, Fox WC, Spiotta AM, Stippler M. A Scoping Review of Burnout in Neurosurgery. Neurosurgery. 2021 Apr;88(5):942-54.

38. Smith RP, Rayburn WF. Burnout in Obstetricians-Gynecologists: Its Prevalence, Identification, Prevention, and Reversal. Obstet Gynecol Clin North Am. 2021 Mar;48(1):231-45.

39. Kirubarajan A, Got T, Park B, Li X, Sobel M. Interventions to Prevent and Treat Burnout in Obstetrics/ Gynaecology: A Scoping Review. J Obstet Gynaecol Can. 2021 Apr;43(4):490-6.

40. Dijxhoorn AQ, Brom L, van der Linden YM, Leget C, Raijmakers NJ. Prevalence of burnout in healthcare professionals providing palliative care and the effect of interventions to reduce symptoms: A systematic literature review. Palliat Med. 2021 Jan;35(1):6-26.

41. Lebares CC, Coaston TN, Delucchi KL, Guvva EV, Shen WT, Staffaroni AM, et al. Enhanced Stress Resilience 
Training in Surgeons: Iterative Adaptation and Biopsychosocial Effects in 2 Small Randomized Trials. Ann Surg. 2021 Mar;273(3):424-32.

42. Smith JM, Boe EA, Will R. Physician Wellness in Orthopedic Surgery: challenges and Solutions. Orthop Clin
North Am. 2021 Jan;52(1):41-52.

43. Bingemann TA, Laubach S. Physician well-being in allergy and immunology: creating a culture of wellness. Ann Allergy Asthma Immunol. 2021 Mar;126(3):219-27. 